\title{
FEATURES
}

\section{Fish, frogs, flies and other fauna in scientific firsts}

\author{
BY SARAH DEWEERDT
}

8 SEPTEMBER 2021

Over the past century, scientists have used a variety of animal models to advance their understanding of the developing brain and autism. Here's a chronology of some of the landmark discoveries involving uncommon autism models.

1916

\section{Notch up:}

Biologist Thomas Hunt Morgan identifies a fruit fly gene called NOTCH that affects wing maturation and is part of a pathway later linked to nervous system development in people.

1920

\section{Heart to heart:}

Austrian scientist Otto Loewi observes parallel activity in two frog hearts suspended in saline, theorizing that a chemical messenger he dubs 'vagusstoff' traveled through the solution - the first identified neurotransmitter. 
1962

\section{Pluripotent possibilities:}

English zoologist John Gurdon replaces the nucleus of a frog egg with a nucleus from an intestinal cell. The resulting embryo successfully develops into a tadpole, demonstrating that mature cells contain all the information needed to create any cell type and kicking off research in induced pluripotent stem cells.

1971

\section{Fly time:}

Biologist Seymour Benzer and a colleague demonstrate that mutations in a fruit fly gene dubbed PER disrupt the fly's circadian rhythm, launching Drosophila melanogaster as a model for the study of how genes control behavior.

1986

\section{Worm wiring:}

Scientists publish the complete wiring diagram of the Caenorhabditis elegans nervous system, paving the way for the extensive use of roundworms in studies of nervous system development.

1990

\section{Elegans experiment:}


Studies show that $C$. elegans is capable of habituation, a simple form of learning that may be disrupted in autism.

2007

\section{Mouse milestone:}

Researchers debut a single-gene mouse model of autism: mice with a mutation in the gene NLGN3.

2010

\section{Inching forward:}

Scientists create one of the first invertebrate models of autism: a strain of $C$. elegans with a faulty version of the roundworm's neuroligin gene NLG1.

2012

\section{Fish tale:}

A zebrafish study shows that multiple genes in the 16p11.2 chromosomal region, which may be deleted or duplicated in autism, play roles in brain development. 


\section{Social space:}

Researchers report that fruit flies in an enclosure tend to keep a reliable amount of space between them, an observation that can be used to investigate how mutations in autism-linked genes affect social behavior.

2015

\section{Eight is enough:}

The complete genome sequence of the California two-spot octopus (Octopus bimaculoides) is published, revealing striking similarities to people in genes involved in nervous system development and function.

2016

\section{Sedated swimmers:}

Zebrafish larvae lacking the autism-linked gene CNTNAP2 are hyperactive at night, but adding estrogen-like compounds to their tank calms the fish - demonstrating a use for the animals in screening potential autism treatments.

2018

\section{Two to tango:}

A study of gene expression in the developing fly eye reveals the importance of interactions between genes in the $16 p 11.2$ region during neurodevelopment. 
2018

\section{Social circuits:}

Researchers identify neurons in the forebrain of zebrafish that support their social interactions, suggesting that zebrafish can be used to probe social brain circuitry.

2019

\section{Picking priorities:}

An analysis of 20 autism-linked mutations in roundworms flags 10 autism candidate genes as worthy of further study, because of their impact on the worm.

2019

\section{Gut feeling:}

Scientists find that food moves through the gut slowly in zebrafish larvae with SHANK3 mutations, providing a clue to the roots of digestive problems associated with autism.

2019

\section{Habit forming:}

The behavior of fruit flies with mutations in autism-linked genes suggests that dozens of these mutations lead to impairments in habituation. 
2019

\section{Stranger danger:}

Like their mouse counterparts, zebrafish lacking the autism-linked gene RELN show little interest in socializing with unfamiliar fish, supporting use of the fish as a model for social difficulties in autism.

2020

\section{Roundworm rodeo:}

A study of more than 27,000 roundworms with mutations in the worm versions of 98 autism-linked genes hints at the function of these genes; many of them affect habituation.

2020

\section{Sound check:}

Zebrafish larvae lacking FMR1, the gene altered in fragile $X$ syndrome, are hypersensitive to sound, suggesting that zebrafish can be used to study sensory processing differences in autism.

2021

\section{Show stopper:}

Reducing the expression of the autism-linked gene FOXP1 in a particular brain region in songbirds 


\section{Spectrum | Autism Research News}

https://www.spectrumnews.org

impairs the birds' ability to memorize songs they hear from their fathers, suggesting a specific role for this gene in language learning.

2021

\section{Fly by night:}

Fruit flies lacking a gene akin to the autism-linked genes CHD7 and CHD8 have fragmented sleep , a problem researchers corrected using an adapted form of 'sleep restriction,' an insomnia treatment.

Cite this article: https://doi.org/10.53053/TIYT8223 